\title{
A customized program for the identification of conserved protein sequence motifs
}

\author{
Mohammad Mian', Jeffrey Talada' , Anthony Klobas' , Stephanie Torres', Yusuf Rasheed' , Hibah Javed' , Zainab Lughmani' \& Reza Forough*,1
}

\begin{abstract}
We searched for viral protein sequences that could be important for tissue tropism. To achieve this goal, human pathogenic viruses were classified according to the tissue they infect (e.g., pulmonary), irrespective of whether they were enveloped or non-enveloped RNA or DNA viruses. Next, we developed an amino acid sequence alignment program and identified the conserved amino acid motif, VAIVLGG, in alphaviruses. The VAIVLGG sequence is located on the structural capsid protein of the chikungunya virus, a mosquito-borne arthrogenic member of the alphaviruses. Capsid protein translocation onto the host cell membrane is a required step for virion budding. Our identified VAIVLGG consensus sequence might potentially be used for developing a pan-vaccine effective against alphaviruses.
\end{abstract}

\section{METHOD SUMMARY}

Viral protein sequences are fed into a battery of rolling hashes of 6-14 length, and amino acid subsequences are performed with a time complexity of $O(n)$. The hashes are the keys in a HashMap with values of the sequence ID and index; the space complexity is $O(n)$. A normal alignment is done on 14 length matches to discover longer matches. The upper bound on the time complexity for the alignment is $O(k 2 * \mathrm{~m})$, where $k$ is the number of viruses containing a matching sequence and $m$ is the length of the longest matching sequence.

\section{KEYWORDS}

alignment software program • alphaviruses - chikungunya virus - CHIKV spike glycoprotein $\cdot$ CHIKV structural capsid protein - Global Virome Project • protein sequence alignment $\cdot$ vaccine development $\cdot$ viral protein homologies

'Biology Program, Science Division, Bellevue College, Bellevue, WA 98007, USA; *Author for correspondence: reza.forough@bellevuecollege.edu

BioTechniques 68:45-47 (January 2020) 10.2144/ btn-2019-0039
We were originally inspired by the recent launch of the Global Virome Project (GVP), a multinational project aimed at discovering new viruses that can potentially infect people or become pandemic [1]. According to the GVP, it is predicted that 1.67 million undiscovered viral species exist in mammals and birds, with roughly half of these unknown viruses possessing zoonotic potential. Our reasoning was that, if the GVP is preparing for the mammoth task of identifying roughly $600,000-$ 800,000 potentially human pathogenic viruses, then there is a need for developing new methods for faster data collection and analysis. In this context, we focused on the following two strategies: developing a new conceptual method of viral classification to provide a better representation of viral pathogenicity and writing customized software to speed up the data retrieval and analysis.

First, we proceeded to prepare a list of all 265 known human pathogenic viruses using the ExPASy ViralZone database [2]. Next, we chose to classify these human pathogenic viruses into subcategories based on the diseases they cause. Thus, each of the 265 human pathogenic viruses was placed into one of the following disease subcategories: fever and joint pain, gastroenteritis, hepatitis, skin lesions, respiratory diseases, encephalitis or hemorrhagic fever, regardless of whether they were RNA or DNA or enveloped or non-enveloped viruses.

We then wrote a customized program to speed up alignments and comparisons among protein sequences of viruses within each subcategory (Figure 1). Our goal was to identify conserved protein sequences within each subcategory with the hope that the identified viral conserved protein motifs might play central roles in determining tissue tropism and successful human host infection. We identified several meaningful conserved protein motifs within each disease subcategory (Figure 2); for example, the CDQMTGILAT and RAFSHLA motifs are located within the viral methyltransferase protein sequence. Methyltransferase is an enzyme important in mRNA capping. Another point worth mentioning is that we used only the ExPASy viral protein sequence database for our study. Our detailed comparison analysis demonstrated that the ExPASy and GenBank viral protein databases are not completely the same (data not shown).

In this manuscript, we have focused on VAIVLGG, a particularly interesting consensus sequence common to many

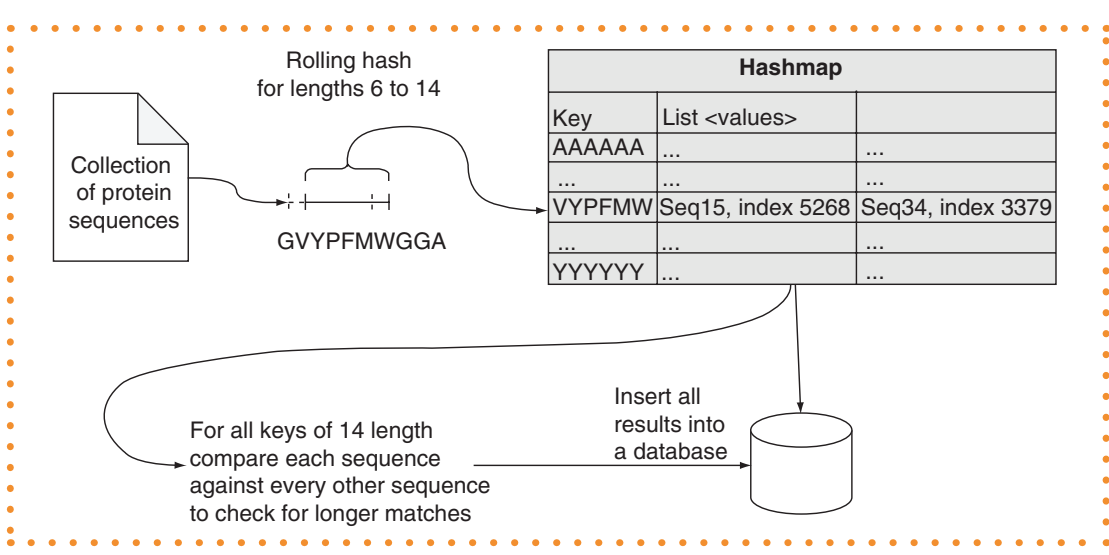

Figure 1. The schematic diagram represents our customized program written in Java to significantly decrease our search time. Using a smaller curated dataset containing only protein sequences from the human pathogenic viruses, we were able to search significantly faster than a query of every $10 \mathrm{~s}$ using BLASTX search. 

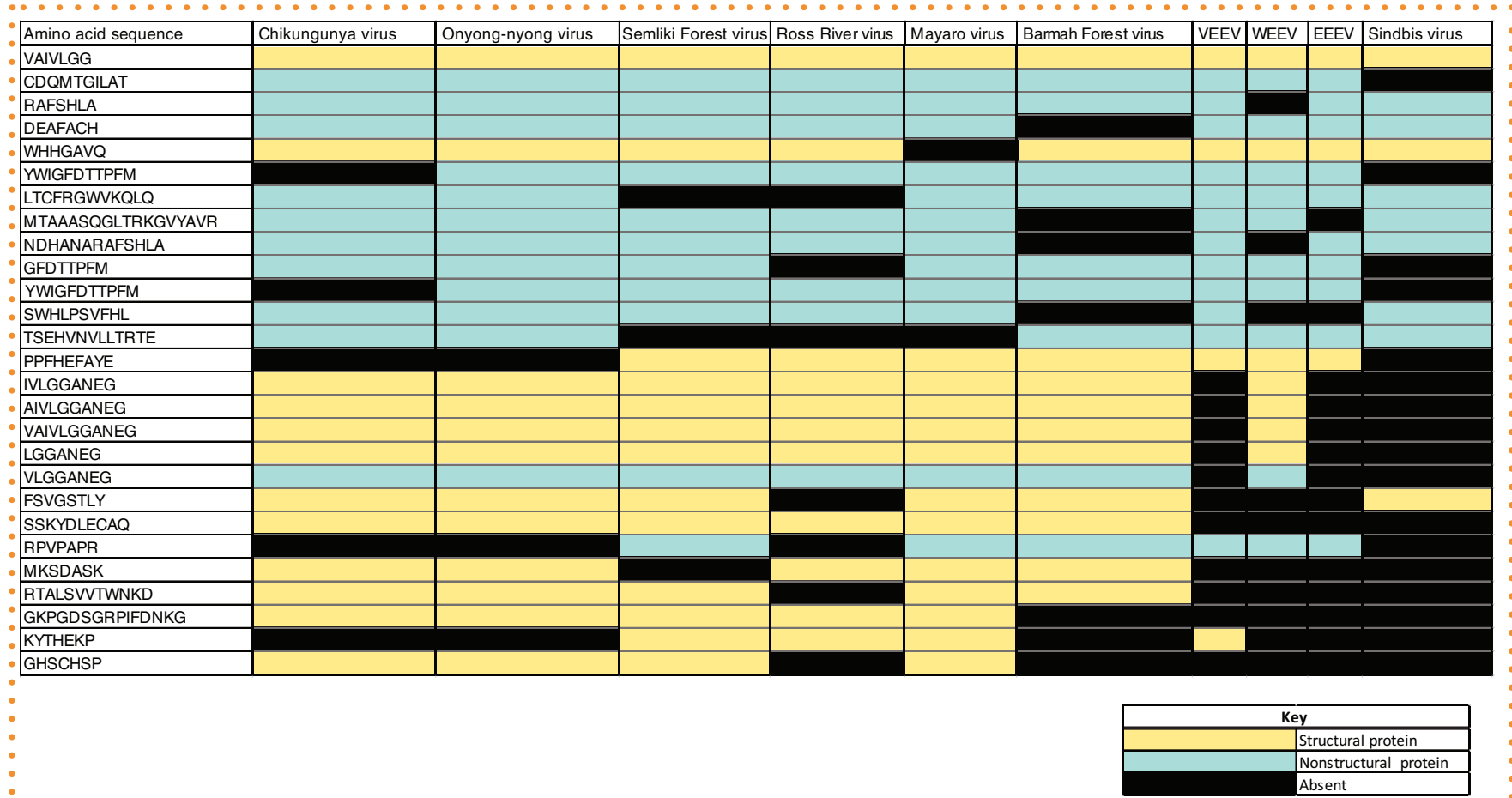

Figure 2. Consensus amino acid motifs identified in alphavirus family members.

different members of alphaviruses known to cause fever and joint pain (Figure 2). Broadly speaking, alphaviruses are divided into 'Old World' and 'New World' alphaviruses. Alphaviruses can cause joint pain and fever leading to arthritis and encephalitis in humans and other animals. Alphaviruses can be transmitted among and within species via mosquito bites. Alphaviruses possess four structural and four non-structural (regulatory) virally coded genes [3]. The VAIVLGG consensus sequence that we identified is located within alphavirus structural proteins [4]. Among all known alphaviruses, special attention is given to chikungunya virus (CHIKV) because of the major epidemic outbreaks that the virus has caused in Africa, Asia and Europe [5]. More recently, CHIKV was reported in a major outbreak in Florida [6]. Mosquitoborne viral pathogens are major concerns when it comes to pandemic outbreaks. Furthermore, currently there is no vaccine available against $\mathrm{CHIKV}$.

We subsequently mapped the VAIVLGG consensus sequence to the structural capsid protein (CP) of CHIKV [7]. It is important to note that the budding mechanism of the infectious alphavirus particles from their host cells involves independent translo- cations of the viral CP and glycoprotein spikes to their host cell plasma membrane, followed by mandatory $\mathrm{CP}$ interaction with the spike protein $[8,9]$. Our further analysis of the human genome also showed that the VAIVLGG sequence is not found in the human proteome. Given the above described findings, we are proposing that the VAIVLGG consensus sequence might potentially be used not only to develop vaccines against CHIKV but also to serve as a single antigen for producing pan-vaccines effective against a wide range of other 'Old World' and 'New World' alphaviruses, including Onyongnyong, Semiliki Forest, Ross River, Mayaro, VEEV, WEEV, EEEV and Sindbis.

New technological advances have provided evidence that there are far more viruses potentially capable of causing human diseases than currently identified. Our described approach will be helpful in identifying common virulence factors in seemingly unrelated human pathogenic viruses, as well as in identifying and characterizing unknown human viral pathogens. The next fundamental step will be the development of preventive vaccines and drugs to fight off the diseases caused by these viruses. A salient feature of our approach is its potential to identify viral protein motifs as potential target sites to aid in the development of pharmaceutical interventions and single-antigen pan-vaccines for the treatment and prevention of these diseases. Other future potential uses of our approach include comparative analyses of non-human infectious viruses to look for motifs that cause certain symptoms, to attack disease vectors or to identify potential mutations that would result in human transmission. The technique could also be extended to studying and identifying target sites in non-viral microbial pathogens such as bacteria, protista and fungi, to name a few.

\section{AUTHOR CONTRIBUTIONS}

$M$ Mian and S Torres performed the viral classification, and M Milan performed the data analysis. J Talada and A Klobas developed the software program, and $J$ Talada wrote the description of the software. Y Rasheed, $\mathrm{H}$ Javed and Z Lughmani helped with the data collection. R Forough supervised the project and wrote the manuscript. All authors read and approved the final manuscript for submission.

\section{ACKNOWLEDGMENTS}

The authors thank Dr Kouros Motamed for his comments on this manuscript. 


\section{FINANCIAL \& COMPETING}

\section{INTERESTS DISCLOSURE}

The authors have no relevant affiliations or financial involvement with any organization or entity with a financial interest in or financial conflict with the subject matter or materials discussed in the manuscript. This includes employment, consultancies, honoraria, stock ownership or options, expert testimony, grants or patents received or pending or royalties.

No writing assistance was utilized in the production of this manuscript.

\section{OPEN ACCESS}

This work is licensed under the AttributionNonCommercial-NoDerivatives 4.0 Unported License. To view a copy of this license, visit http://creativecommons.org/licenses/ by-nc-nd/4.0/

\section{REFERENCES}

1. Carroll D, Daszak $\mathrm{P}$, Wolfe $\mathrm{N}$ et al. The global virome project. Science 359, 872-874 (1998).

2. ExPASy. ViralZone. https://viralzone.expasy.org/678

3. Strauss JH, Strauss EG. The alphaviruses: gene expression, replication, evolution. Microbiol. Rev. 58, 491-562 (1994).

4. Voss JE, Vaney MC, Duquerroy S et al. Glycoprotein organization of chikungunya virus particles revealed by $x$-ray crystallography. Nature $468(7324), 709-712$ (2010).

5. Wahid B, Ali A, Rafique S, Idrees M. Global expansion of chikungunya virus: mapping the 64 -year history. Int. J. Infect. Dis. 58, 1-118 (2017).

6. Alto BW, Wiggins K, Eastmond B, Velez D, Lounibos LP, Lord CC. Transmission risk of two chikungunya lineages by invasive mosquito vectors from Florida and the Dominican Republic. PLoS Negl. Trop. Dis. 11(7), e0005724 (2017).

7. Sharma R, Kesari P, Kumar P, Tomar S. Structure-function insights into chikungunya virus capsid protein: small molecules targeting capsid hydrophobic pocket. Virology 515, 223-234 (2018).

8. Suomalainen M, Liljeström P, Garoff H. Spike protein-nucleocapsid interactions drive the budding of alphaviruses. J. Virol. 66(8), 4737-4747 (1992).

9. Jose J, Snyder JE, Kuhn RJ. A structural and functional perspective of alphavirus replication and assembly. Future Microbiol. 4(7), 837-856 (2009). 